Bangladesh J. Zool. 49 (2): 289-300, 2021

ISSN: 0304-9027

eISSN: $2408-8455$

\title{
MARKET INFRASTRUCTURE AND SPECIES AVAILABILITY OF FOUR WHOLESALE FISH MARKETS IN DHAKA CITY, BANGLADESH
}

\author{
Jannatul Ferdhosi Shikha and Rumana Tasmin* \\ Department of Zoology, Faculty of life and Earth Science. Jagannath University, \\ Dhaka-1100, Bangladesh
}

\begin{abstract}
The study was conducted on species availability and infrastructure analysis of four different wholesale fish markets in Dhaka City. Two markets were selected from Dhaka South City Corporation, Showari Ghat and Jatrabari fish market, and two from Dhaka North City Corporation, Karwan Bazar and Abdullahpur fish market. The investigation was carried out from November 2018 to October 2019. Questionnaire based survey, FGDs (Focus Group Discussion) and KIIs (Key Informant Interviews) were conducted to get quantitative and qualitative data for present study. A total number of 62 fishes and 6 crustacean species were recorded during the study period. Among all species, 39 freshwater, 10 marine and 13 exotic fishes were recorded. Infrastructure facilities were very poor for all four markets in the matter of hygiene and quality control. Fish shades were not enough, improper sanitation and drainage system indicates the possibilities of disease transmission and deteriorate fish quality. Electricity and water supplies were good but no system was present to determine the distillation of those supplied water. Thus, infrastructural condition of the markets does not support a hygienic condition for fish marketing to provide quality fish and fish byproducts. The study highlighted the need for national initiatives, awareness building, and law implementation for improving the wholesale markets infrastructure to a minimum standard so that fish trade could provide good quality to the people of Dhaka City.
\end{abstract}

Keywords: Species availability, Infrastructure, Wholesale fish market, Hygiene.

\section{INTRODUCTION}

Bangladesh has a vast freshwater and marine ecosystem with rich species biodiversity. Fisheries commodities are accumulated from different sources of water bodies, such as river, beel, pond, gher, estuaries and sea, and these fishes are transferred to local markets via different intermediaries and channels (Ali et al. 2004). Though Bangladesh is a small country of South Asia but it has a great resource of fish and other aquatic animals. Inland aquatic habitats of

*Author for corresponding: tasminrumana79@gmail.com

C2021 Zoological Society of Bangladesh DOI: https://doi.org/10.3329/bjz.v49i2.56265 
Bangladesh are rich in faunal diversity containing at least 265 species of finfish, 63 species of prawn, and several species of turtles, tortoises, freshwater mussels and other living aquatic organisms (Rahman 2005). Bangladesh is also rich in marine fishes having 475 marine finfish and 36 Marine shrimp species (DoF 2013). In 2017-18, fisheries sector contributed 3.57\% to national GDP and $25.30 \%$ to the agricultural GDP and $1.5 \%$ to foreign exchange earnings by exporting fish and fish products (DoF 2018). Buriganga, Turag, Dhaleshwari and Shitalakshya are bounded the Dhaka city which is the capital of Bangladesh. According to Bangladesh Bureau of Statistics, it is one of the largest and most populated cities in the country with a population of 21 million (Islam et al. 2015). Fish is the primary protein source in Bangladeshi diet contributing about $60 \%$ of total animal protein (DoF 2018; Jessica et al. 2015); According to BBS report, per capita fish consumption in the country reaches $62.58 \mathrm{gm}$, which is higher than their daily protein demand (60 gm) (BBS 2017). In 2014-2015, total fishery production of Bangladesh was 3,684,245 metric tons, of which $1,023,991$ metric tons was obtained from inland capture fisheries, 2,060,408 metric tons from inland aquaculture and 599,846 metric tons from marine water production (FRSS 2016). There are several fish markets found in Dhaka city where fish accepted from all over the country especially from the southern part of Bangladesh. Availability of fish to consumers at the right time and in the right place requires an effective marketing system (Bahadur 2004).

In most developing countries, main constraints of fish marketing were related to infrastructure, plant management and institutional management aspect (Alam et al. 2010). Infrastructure of the fish markets should be hygienic and suitable for the protection of any perishable condition in a fish market (Cuter et al. 2005). Infrastructure development of fish markets of Dhaka city is an imperative issue to get quality fish and prevent disease transmission from unhygienic condition.

Therefore, overall objectives of this study was to identify the present infrastructure condition and survey the available fish species on those wholesale markets in Dhaka city from the point of view on hygiene and environmental aspect.

\section{MATERIAL AND METHODS}

Study period: The study was carried out for a period of one year from November, 2018 to October, 2019 on four wholesale fish markets in Dhaka city.

Study area: There were many fish markets in and around the Dhaka city, among them total four wholesale fish markets were selected for this study. Two fish markets are Showari Ghat and Jatrabari from Dhaka South and others are 
Abdullahpur fish market and Karwan Bazaar from Dhaka North City Corporation area (Figure 1).

Methods of data collection and analysis: A combination of eye observation and survey through questionnaire were adopted for this study to understand the overall infrastructural situation and available fish species in four fish markets. Structured interview schedules were used to collect information about supply condition of ice, water and electricity, sanitation facilities and major constrains from selected wholesale markets through FGD (focus group discussion) and KIIs (Key Informant Interviews) with operators and officials of the markets, members of trader's associations and wholesalers. A total of 16 FGDs (6 in Jatrabari fish market, 4 in Showari Ghat fish market, 3 in Karwan bazaar and 3 in Abdullapur fish market) were conducted where each group size of FGD was 4 to 9 person. Cross-check interviews were conducted with key informant's interviews and each interview last on average 2 to 5 minutes. The data were analyzed using Microsoft Excel software. Research tools to collect detail data are presented in Table 1.

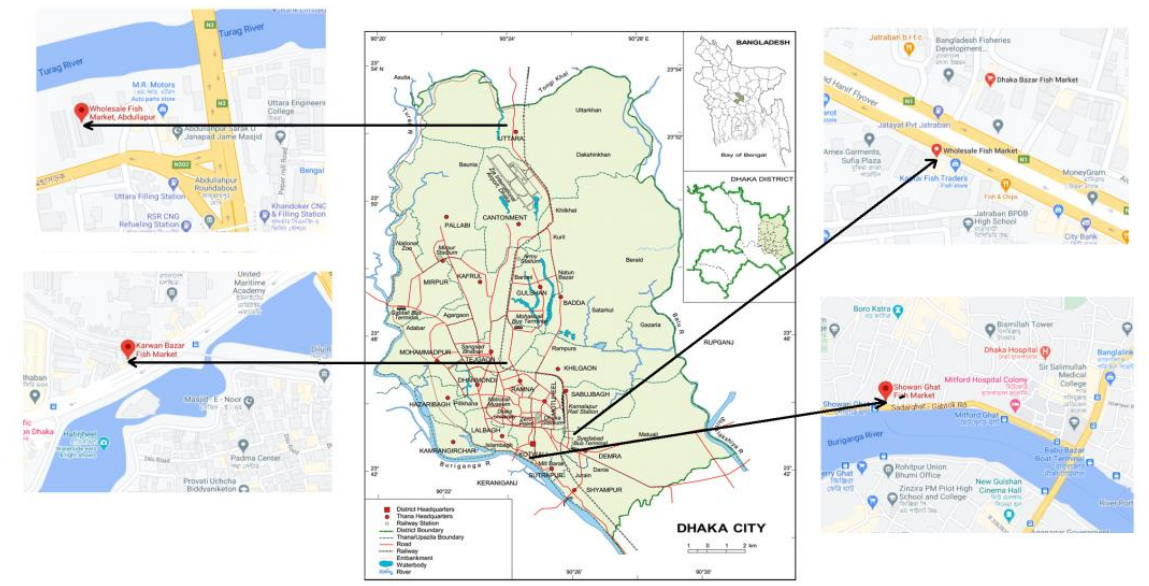

Fig. 1. Map of study area in Dhaka city.

Table 1. Methodological tools used for data collection

\begin{tabular}{lll}
\hline $\begin{array}{l}\text { Data Collection } \\
\text { Techniques }\end{array}$ & \multicolumn{1}{c}{ Study Areas } & Collected Information \\
\hline $\begin{array}{l}\text { Questionnaire interview } \\
\text { and direct eye } \\
\text { observation }\end{array}$ & $\begin{array}{l}\text { Showari Ghat, Jatrabari, } \\
\text { Karwan bazaar and } \\
\text { Abdullahpur fish market }\end{array}$ & $\begin{array}{l}\text { About fish species, building } \\
\text { information, environmental } \\
\text { condition, drainage system, } \\
\text { roof and floor condition ice } \\
\text { Sanitation facility, ice, } \\
\text { electricity, water supply } \\
\text { and major constrains }\end{array}$ \\
\hline
\end{tabular}


Table2. The comparative scenario of present infrastructural condition in four fish markets

\begin{tabular}{|c|c|c|c|c|}
\hline Physical facilities & $\begin{array}{l}\text { Showari Ghat } \\
\text { fish market }\end{array}$ & $\begin{array}{l}\text { Jatrabarifish } \\
\text { market }\end{array}$ & $\begin{array}{l}\text { Karwan } \\
\text { Bazaar }\end{array}$ & $\begin{array}{l}\text { Abdullapur fish } \\
\text { market }\end{array}$ \\
\hline Quality of floor & $\begin{array}{l}\text { Some floor are } \\
\text { cemented } \\
\text { some are mud } \\
\text { cracked and } \\
\text { rough }\end{array}$ & $\begin{array}{l}\text { Some floors are } \\
\text { cemented and } \\
\text { smooth, some are } \\
\text { mud and cracked } \\
\text { in different places }\end{array}$ & $\begin{array}{l}\text { Concreted, } \\
\text { rough and } \\
\text { somewhere } \\
\text { cracked }\end{array}$ & $\begin{array}{l}\text { Some floors are } \\
\text { cemented and } \\
\text { smooth, some are } \\
\text { mud and cracked } \\
\text { in different places }\end{array}$ \\
\hline Storage facility & Present & Present & Absent & Absent \\
\hline Ice factory & Two & Four & Absent & Two \\
\hline Electricity supply & Present & Present & Present & Present \\
\hline Water supply & Present & Present & Present & Present \\
\hline $\begin{array}{l}\text { Market owned } \\
\text { toilet }\end{array}$ & One & Absent & present & Six \\
\hline $\begin{array}{l}\text { Fish dumping } \\
\text { place }\end{array}$ & $\begin{array}{l}\text { Mud built } \\
\text { floor and } \\
\text { basket }\end{array}$ & $\begin{array}{l}\text { On the platform } \\
\text { and basket }\end{array}$ & $\begin{array}{l}\text { On the } \\
\text { platform and } \\
\text { basket }\end{array}$ & $\begin{array}{l}\text { On the platform, } \\
\text { mud built floor and } \\
\text { basket }\end{array}$ \\
\hline Drainage system & $\begin{array}{l}\text { The floors } \\
\text { don't have a } \\
\text { clear slope to } \\
\text { a drain }\end{array}$ & $\begin{array}{l}\text { The floors have a } \\
\text { clear sloop to a } \\
\text { drain }\end{array}$ & $\begin{array}{l}\text { The floors } \\
\text { have a clear } \\
\text { sloop to a } \\
\text { drain }\end{array}$ & $\begin{array}{l}\text { The floors do not } \\
\text { have a clear slope } \\
\text { to a drain }\end{array}$ \\
\hline $\begin{array}{l}\text { Protective } \\
\text { enclosure to } \\
\text { prevent entry of } \\
\text { pests }\end{array}$ & Absent & $\begin{array}{l}\text { Some parts, 'Yes' } \\
\text { and some parts, } \\
\text { 'No' }\end{array}$ & Absent & Absent \\
\hline Roof condition & $\begin{array}{l}\text { Some made } \\
\text { with plastic } \\
\text { and some } \\
\text { with tin shade }\end{array}$ & $\begin{array}{l}\text { Some place } \\
\text { concreted and } \\
\text { some with tin } \\
\text { shade }\end{array}$ & $\begin{array}{l}\text { Some made } \\
\text { with plastic } \\
\text { and some } \\
\text { with tin } \\
\text { shade }\end{array}$ & $\begin{array}{l}\text { Some made with } \\
\text { plastic and some } \\
\text { with tin shade }\end{array}$ \\
\hline
\end{tabular}

\section{RESULTS AND DISCUSSION}

Infrastructural condition: Infrastructure facilities were very poor for all four markets. Fish shades were not enough, lack of hygienic management indicates the possibilities of disease transmission, deteriorates fish quality which could be great issues for public health especially in any pandemic situation. Electricity and water supplies were good but no system was present to determine the distillation of those supplied water. This infrastructural condition of the markets does not support a hygienic condition for fish marketing to provide quality fish and fish byproducts (Table 2).

Seasonal variation of available fish species in four markets: On the basis of total species availability all the year round, mostly abundant was in rainy season and it was 45 percent and others 33 and 22 percent in winter and summer season, respectively (Figure 2).

Freshwater species: Among 39 species of freshwater fishes, koi (Anabas testudineus), Taki (Channa punctata), Ilish (Tenualosa ilisha), Rui (Labeo rohita),Shing (Heteropneustes fossilis), Catla (Catla catla), Mrigel (Cirrhinus 
cirrhosus), Pangas (Pangasius pangasius) found mostly in the all four fish markets (Table 3).

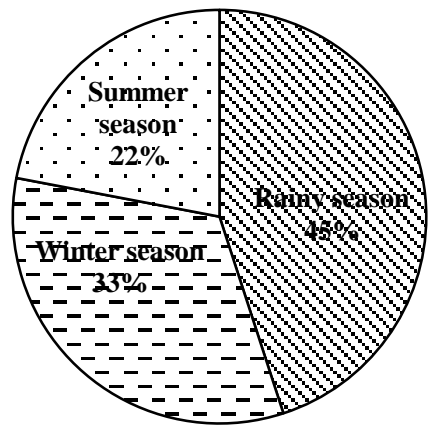

Fig. 2. Availability of fish species in different season from all markets.

Table 3. List of freshwater fish species available in different fish markets in Dhaka City

\begin{tabular}{|c|c|c|c|c|c|}
\hline Family & Local Name & Common Name & $\begin{array}{l}\text { Scientific } \\
\text { Name }\end{array}$ & $\begin{array}{l}\text { Seasonal } \\
\text { Abundance }\end{array}$ & Market Name \\
\hline Anabantidae & Koi & Climbing perch & $\begin{array}{l}\text { Anabas } \\
\text { testudineus }\end{array}$ & TY & All markets \\
\hline Anguillidae & Bao Baim & Giant Mottled eel & $\begin{array}{l}\text { Anguilla } \\
\text { bengalensis }\end{array}$ & WS & $\begin{array}{l}\text { Jatrabari, Abdullahpur, } \\
\text { karwan Bazaar fish } \\
\text { market }\end{array}$ \\
\hline \multirow[t]{2}{*}{ Ambassidae } & Chanda & $\begin{array}{l}\text { Elongate glass- } \\
\text { perchlet }\end{array}$ & Chanda nama & WS & All markets \\
\hline & Kata chanda & $\begin{array}{l}\text { Himalayan glassy- } \\
\text { perchlet }\end{array}$ & $\begin{array}{l}\text { Parambasssis } \\
\text { baculis }\end{array}$ & SS & All markets \\
\hline \multirow[t]{4}{*}{ Bagridae } & Tengra & $\begin{array}{l}\text { Striped dwart } \\
\text { catfish }\end{array}$ & Mystus vittatus & TY & All markets \\
\hline & Golsha Tengra & Gangeticmystus & Mystus bleekeri & TY & $\begin{array}{l}\text { Jatrabari, Abdullapur, } \\
\text { karwan Bazaar fish } \\
\text { market }\end{array}$ \\
\hline & Ayre & $\begin{array}{l}\text { Long-whiskered } \\
\text { catfish }\end{array}$ & Sperata aor & RS & All markets \\
\hline & Rita & Rita & Rita rita & WS & $\begin{array}{l}\text { Jatrabari, karwan Bazaar } \\
\text { fish market }\end{array}$ \\
\hline Belonidae & Kaikka & Needle fish & $\begin{array}{l}\text { Xenentodon } \\
\text { cancila }\end{array}$ & WS & All markets \\
\hline \multirow[t]{3}{*}{ Chnnidae } & Shol & $\begin{array}{l}\text { Striped } \\
\text { snakehead }\end{array}$ & Channa striatus & WS & All markets \\
\hline & Gajar & Giant snakehead & $\begin{array}{l}\text { Channa } \\
\text { marulius }\end{array}$ & SS & $\begin{array}{l}\text { Jatrabatri, karwan } \\
\text { Bazaar, Showari Ghat } \\
\text { fish market }\end{array}$ \\
\hline & Taki & $\begin{array}{l}\text { Spotted } \\
\text { snakehead }\end{array}$ & $\begin{array}{l}\text { Channa } \\
\text { punctata }\end{array}$ & TY & All markets \\
\hline Clariidae & Magur & $\begin{array}{l}\text { Air breathing } \\
\text { catfish }\end{array}$ & $\begin{array}{l}\text { Clarias } \\
\text { batrachus }\end{array}$ & WS & All markets \\
\hline
\end{tabular}




\begin{tabular}{|c|c|c|c|c|c|}
\hline Family & Local Name & Common Name & $\begin{array}{l}\text { Scientific } \\
\text { Name }\end{array}$ & $\begin{array}{l}\text { Seasonal } \\
\text { Abundance }\end{array}$ & Market Name \\
\hline \multirow[t]{3}{*}{ Clupeidae } & llish & Hilsa & $\begin{array}{l}\text { Tenualosa } \\
\text { ilisha }\end{array}$ & TY & All markets \\
\hline & Kachki & Ganga river & Corica soborna & WS & All markets \\
\hline & Chapila & Indian river shad & Gudusia chapra & WS & All markets \\
\hline \multirow[t]{9}{*}{ Cyprinidae } & Rui & Rohu & Labeo rohita & TY & All markets \\
\hline & Catla & Catla & Catla catla & TY & All markets \\
\hline & Mrigel & Mrigal & $\begin{array}{l}\text { Cirrhinus } \\
\text { cirrhosus }\end{array}$ & TY & All markets \\
\hline & Kalibaus & Black rohu & Labeo calbasu & TY & All markets \\
\hline & Bhangonbata & Bata labeo & Labeo bata & SS & All markets \\
\hline & Sarpunti & Olive barb & Puntius sarana & TY & All markets \\
\hline & Cholapunti & Swamp barb & Puntius chola & SS & All markets \\
\hline & Mola & Molacarplet & $\begin{array}{l}\text { Amblypharyngo } \\
\text { do mola }\end{array}$ & SS & All markets \\
\hline & Dhela & Cotio & Rohtee cotio & WS & All markets \\
\hline Gobiidae & Bele & Tank goby & $\begin{array}{l}\text { Glossogobius } \\
\text { giuris }\end{array}$ & WS & Jatrabari fish market \\
\hline Heteropneustidae & Shing & Stinging catfish & $\begin{array}{l}\text { Heteropneustes } \\
\text { fossilis }\end{array}$ & TY & All markets \\
\hline \multirow[t]{2}{*}{ Mastacembelidae } & Baim & Tire-track spiny eel & $\begin{array}{l}\text { Mastacembelus } \\
\text { armatus }\end{array}$ & TY & $\begin{array}{l}\text { Jatrabari, Showari Ghat } \\
\text { fish market }\end{array}$ \\
\hline & Tara baim & $\begin{array}{l}\text { One stripe } \\
\text { spiny eel }\end{array}$ & $\begin{array}{l}\text { Macrognathus } \\
\text { aculeatus }\end{array}$ & TY & Jatrabari fish market \\
\hline Nandidae & Bheda & Mud perch & Nandus nandus & SS & All markets \\
\hline \multirow[t]{2}{*}{ Notopteridae } & Chital & $\begin{array}{l}\text { Humped } \\
\text { featherback }\end{array}$ & $\begin{array}{l}\text { Notopterus } \\
\text { chitala }\end{array}$ & RS & All markets \\
\hline & Foli & Grey featherback & $\begin{array}{l}\text { Notopterus } \\
\text { notopterus }\end{array}$ & RS & $\begin{array}{l}\text { Jatrabari, karwan Bazaar } \\
\text { fish market }\end{array}$ \\
\hline \multirow[t]{2}{*}{ Osphronemidae } & $\begin{array}{l}\text { Khalisha/Khail } \\
\text { sha }\end{array}$ & Giant gourami & Colisa fasciatus & WS & All markets \\
\hline & LalKhalisha & Red gourami & Colisa Ialia & WS & $\begin{array}{l}\text { Jatrabari, Abdullapur fish } \\
\text { market }\end{array}$ \\
\hline Pangasidae & Pangas & Yellowtail catfish & $\begin{array}{l}\text { Pangasius } \\
\text { pangasius }\end{array}$ & TY & All markets \\
\hline \multirow[t]{3}{*}{ Siluridae } & Boal & Freshwater shark & Wallagaattu & TY & All markets \\
\hline & Pabda & $\begin{array}{l}\text { Indian butter } \\
\text { catfish }\end{array}$ & Ompok pabda & TY & All markets \\
\hline & Madhupabda & Pabo catfish & Ompok pabo & TY & $\begin{array}{l}\text { Jareabari, karwan } \\
\text { bazaar, Showari Ghat } \\
\text { fish market }\end{array}$ \\
\hline Sisoridae & Baghair & Devil catfish & $\begin{array}{l}\text { Bagarius } \\
\text { bagarius }\end{array}$ & TY & Jatrabari fish market \\
\hline Synbrachidae & Kuicha & Kuchia & $\begin{array}{l}\text { Monopterus } \\
\text { cuchia }\end{array}$ & TY & Jatrabari fish market \\
\hline
\end{tabular}

$\mathrm{NB}: \mathrm{TY}=$ throughout the year, $\mathrm{WS}=$ winter season, $\mathrm{SS}=$ summer season, $\mathrm{RS}=$ rainy season

Species availability in different fish market: A total number of 62 fish species and six crustacean species were recorded during the study period. From all species, there were 39 species from freshwater and 10 species from marine, and 13 were exotic fish.

Marine species: Loitta (Harpadon nehereus), Poa (Pama pama), Lalpoa (Johniusargentatus) found mostly in the four market throughout the year. Other 
fish species also found with great market demand. Now- a-days marine fish species are becoming more popular for their nutritive value (Table 4).

Table 4. List of marine fish species available in different fish markets in Dhaka City

\begin{tabular}{|c|c|c|c|c|c|}
\hline Family & $\begin{array}{l}\text { Local } \\
\text { Name }\end{array}$ & Common Name & Scientific Name & $\begin{array}{l}\text { Seasonal } \\
\text { Abundance }\end{array}$ & Market Name \\
\hline Centropomidae & Bhetki & Bhetki & Lates calcarifer & TY & $\begin{array}{l}\text { Jatraabari, karwan } \\
\text { Bazaar fish market }\end{array}$ \\
\hline Engraulidae & Phasa & Hairpin anchovy & Setipinna taty & SS & Jatrabari fish market \\
\hline Harpadontidae & Loitta & Bombay duck & Harpadon nehereus & TY & All markets \\
\hline \multirow{3}{*}{ Sciaenidae } & Poa & Pama & Pama pama & TY & All markets \\
\hline & Lalpoa & Silver jew & Johnius argentatus & TY & All markets \\
\hline & Sadapoa & Silver jew & Otolithes argentatus & TY & All markets \\
\hline Scombridae & Mackerel & Indian mackerel & $\begin{array}{l}\text { Rastrelliger } \\
\text { kanagurta }\end{array}$ & TY & $\begin{array}{l}\text { Jatraabari, karwan } \\
\text { Bazaar, abdullapur } \\
\text { fish market }\end{array}$ \\
\hline \multirow[t]{2}{*}{ Stromatidae } & $\begin{array}{l}\text { Rup } \\
\text { chanda }\end{array}$ & Chinese pomfret & Pampus chinensis & TY & All markets \\
\hline & Folichanda & Silver pomfiret & Pampus argenteus & WS & $\begin{array}{l}\text { Jatraabari, karwan } \\
\text { Bazaar fish market }\end{array}$ \\
\hline Tunnidae & Tuna & Born maittya & Euthynnus affinis & WS & All markets \\
\hline
\end{tabular}

Crustacean species: Gura chingri (Nematopalaernon tenuipes), Golda chingri (Macrobrachium rosenbergii), Bagda chingri (Penaeus monodon) found mostly in the four market throughout the year (Table 5).

Exotic Species: Among 13 species of exotic species, Tilapia (Oreochromis mossambicus),African catfish (Clarias gariepinus),Carpu (Cyprinus carpio),Thai pungus (Pangasius hypophthalmus), Silver carp (Hypophthalmichthys molitrix),Thai sarputi (Barbonymus gonionotus) found mostly in the all four fish markets (Table 6). All species were found year round with high demand due to their availability and low cost in the market.

Table 5. List of crustacean's species available in different fish markets in Dhaka City

\begin{tabular}{llllll}
\hline Family & Local Name & Common Name & Scientific Name & $\begin{array}{l}\text { Seasonal } \\
\text { Abundance }\end{array}$ & $\begin{array}{l}\text { Market } \\
\text { Name }\end{array}$ \\
\hline Palaemonidae & Gurachingri & Spider prawn & Nematopalaernon tenuipes & TY & All markets \\
& Golda chingri & Fresh water prawn & Macrobrachium rosenbergii & TY & All markets \\
& Chatka chingri & Monsoon river & Macrobrachium alcolmsonii & TY & All markets \\
& & prawn & & & \\
Penaeidae & Horina chingri & Brown shrimp & Metapenaeusmonoceros & TY & All markets \\
& Bagda chingri & Giant tiger shrimp & Penaeus monodon & TY & All markets \\
& Chaka chingri & Indian white shrimp & Penaeus indicus & TY & All markets \\
\hline
\end{tabular}

$\mathrm{TY}=$ throughout the year 
Table 6. List of exotic species available in different fish markets in Dhaka City

\begin{tabular}{|c|c|c|c|c|c|}
\hline Family & $\begin{array}{l}\text { Local } \\
\text { Name }\end{array}$ & $\begin{array}{l}\text { Common } \\
\text { Name }\end{array}$ & $\begin{array}{l}\text { Scientific } \\
\text { Name } \\
\end{array}$ & $\begin{array}{l}\text { Seasonal } \\
\text { Abundance }\end{array}$ & $\begin{array}{l}\text { Market } \\
\text { Name }\end{array}$ \\
\hline \multirow[t]{3}{*}{ Cichlidae } & Tilapia & $\begin{array}{l}\text { Mozambique } \\
\text { tilapia }\end{array}$ & $\begin{array}{l}\text { Oreochromis } \\
\text { mossambicus }\end{array}$ & TY & All markets \\
\hline & Nilotica & Nile cichlid & $\begin{array}{l}\text { Oreochromis } \\
\text { niloticus }\end{array}$ & TY & All markets \\
\hline & Lal Tilapia & Red tilapia & $\begin{array}{l}\text { Oreochromis } \\
\text { niloticus }\end{array}$ & TY & All markets \\
\hline Clariidae & $\begin{array}{l}\text { African } \\
\text { catfish }\end{array}$ & $\begin{array}{l}\text { North } \\
\text { African } \\
\text { catfish }\end{array}$ & $\begin{array}{l}\text { Clarias } \\
\text { gariepinus }\end{array}$ & TY & All markets \\
\hline \multirow[t]{8}{*}{ Cyprinidae } & $\begin{array}{l}\text { Mirror } \\
\text { carp }\end{array}$ & Mirror carp & $\begin{array}{l}\text { Cyprinus carpio } \\
\text { var } \\
\text { speculararis }\end{array}$ & TY & All markets \\
\hline & Scale carp & Scale carp & $\begin{array}{l}\text { Cyprinus carpio } \\
\text { var nudus }\end{array}$ & TY & All markets \\
\hline & Carpu & $\begin{array}{l}\text { Common } \\
\text { carp }\end{array}$ & $\begin{array}{l}\text { Cyprinus carpio } \\
\text { var communis }\end{array}$ & TY & All markets \\
\hline & Silver carp & Silver carp & $\begin{array}{l}\text { Hypophthalmic } \\
\text { hthys molitrix }\end{array}$ & TY & All markets \\
\hline & $\begin{array}{l}\text { Bighead } \\
\text { carp }\end{array}$ & Bighead carp & $\begin{array}{l}\text { Aristichthys } \\
\text { nobilis }\end{array}$ & TY & All markets \\
\hline & Black carp & Black carp & $\begin{array}{l}\text { Mylopharyngod } \\
\text { on piceus }\end{array}$ & TY & All markets \\
\hline & $\begin{array}{l}\text { Thai } \\
\text { sarputi }\end{array}$ & Silver barb & $\begin{array}{l}\text { Barbonymus } \\
\text { gonionotus }\end{array}$ & TY & All markets \\
\hline & Grass carp & Grass carp & $\begin{array}{l}\text { Ctenopharyngo } \\
\text { don idella }\end{array}$ & TY & All markets \\
\hline Pangasiidae & $\begin{array}{l}\text { Thai } \\
\text { pungus }\end{array}$ & Big-catfish & $\begin{array}{l}\text { Pangasius } \\
\text { hypophthalmus }\end{array}$ & TY & All markets \\
\hline
\end{tabular}

In previous study 71 freshwater species from 25 families from Dhaka (Bhuiyan 1964); 65 species from 29 families from Feni (Yeasmin et al. 2016) were recorded. Another study was conducted by Chowdhury and Iqubal (2007) and they recorded 98 inland and marine water fishes along with crustaceans. Rahman (2005) recorded 260 species in Mymensingh town which is much higher rate according to Dhaka city. According to Aktar et al. (2013), a total 71 to 43 species were recorded from different markets of Noakhali district in Bangladesh. Based on recorded data it was found that species number is getting lower than the previous study and notable popularity of cultured freshwater and exotic fishes were observed almost all the fish markets. Decreasing of indigenous species in the markets may be due to habitat loss, pollution in the environment and high cost to meet the consumers demand (Aziz et al. 2021).

The present infrastructural condition of the markets in Dhaka City is still poorly developed in term of landing, fish storage facility, preserving, transport, sanitation, water supply and retail facilities. Quality of floor of all fish markets were more or less same in nature, floors were cemented or mud and in some 
parts cracked and rough. Storage facility present in Showari Ghat and Jartabari fish markets but those were not sufficient with the number of retailers of those fish markets. Karwan Bazar is an iconic wholesale market in the entire Dhaka City and situated in the middle of the city but this fish market didn't have any storage facility. Ice factory also absent in Karwan Bazar fish market but rest three markets have. Electricity and water supply was present to the all markets but sanitation facility was very poor in all markets. Showari Ghat and Abdullahpur fish markets had market owned toilets but was not much hygienic.

There was no permanent protective enclose found in the study period in the four fish markets only Jatrabari fish markets had some shade in some parts as protective enclose but rest of the three markets didn't have any measure to prevent the entry of unwanted animals or pests. Fish mainly dumped on the platform in all markets. Drainage system was very poor and roof condition was not well developed. Roof mainly found as cemented or plastic shade or tin shade which was not fully cover the protection from any environmental hazard such as heavy rain, storm and high temperature.

According to Rahman et al. (2009), marketing costs become high if marketing functions are not performed efficiently due to functional difficulties such as poor roads or transportation, inadequate storage facility leading to losses; poor handling or lack of knowledge may leads to decrease fish freshness, possibility to transmit many diseases etc. Fish is very sensitive and perishable food items which need a lot of care at the time of marketing. Any kind of miss handling of fishes may occur a great loss of the fisherman and make issues regarding public health. After observing the markets, it was revealed that there was no significant changes occurred in comparison with previous studies (Hussain et al. 1995; Rahman 1997; Hasan et al. 2014). However, considering the previous and present investigations it is clear that the infrastructural condition of wholesale fish markets are still inadequate over long time and unhygienic may cause serious threats to get quality fish and public health issues.

Recommendations: After completion of successful research on four major fish markets of Dhaka city, present study suggests few recommendations for further development of markets through establishment of modern facilities to ensure public health and in case of safety measures;

- Improvement of fish transportation system; such as freezing van for dead fish, follow scientific guidelines for transporting live fish with proper space, oxygenation, aeration in open truck/tank. (more details, Rajts and Shelley, 2020)

- Focus on safe handling hygienically from farm gate to consumer which will help to keep the fish with high price and safe to eat. 
- Priority should be given on proper sanitation system, hygienic condition, drainage and ensure continuous water supply at each major market in Bangladesh.

- Establishment of more ice plants, cold-storage and preservation facilities and improvement of roof or shades.

- Government regular monitoring and strict law implementation must need to control any illegal movement related to human health hazard

- To get the quality and nutritive value of fish, must need to educate farmers, processor and consumer about handling method of aquatic products.

\section{CONCLUSION}

Fish marketing plays an important role in the economy of Bangladesh, contributing to increased fish production on the basis of consumer demand, diversification of the economy, increased employment opportunities. On the other hand, fish market must maintain a high standard of public health hygiene and it will need to comply with national public health regulations e,g,. Food Safety Act, 2013 (BFSA 2019). Fish markets are one of the crucial places which facilitate trade of most perishable food item like fish, but infrastructural facilities are still not satisfactory from the point of view about food safety and environmental aspects. Therefore making collaboration among the scientists of research institutes and universities with fisheries sector in the matter of quality control, disease transmission, breeding and culture of threatened natural fish, introduce new species and provision of governmental, institutional and banking assistance including insurance facilities to make people interest who are directly involve with this profession to encourage and introducing new innovative techniques will boost up the popularity and production of fish. Thus, considering human health as a major concern communication among government, NGOs, stakeholders, policymakers, researcher need to work together to update and upgrade a healthy fish market which would be able to meet the consumer's satisfaction at both national and international level.

\section{LITERATURE CITED}

Aktar N, Islam M.R., Hossain M.B., Rahman M. 2013. Fish species availability and marketing system of fish in different markets of Noakhali district in Bangladesh. World Appl. Sci. J., 22(5): 616624.

Alam J.M., Yasmin R., Rahman A., Nahar N., Pinky I.N. and Hasan M. 2010. A Study on Fish Marketing System in Swarighat, Dhaka, Bangladesh, Nature and Science; 8(12): 96-103. 
Ali M.Y., Salim, G.M., Mannan M.A., Rahman M.M., Sabbir W. and Mursida A. 2004. Fish species availability observed in the fish landing centers of Khulna district in Bangladesh. J. Biol. Sci. 4(5): 575-580.

Aziz M.S.Bin, Hasan N.A., Mondol M.M.R., Alam M.M., Haque M.M. 2021. Decline in fish species diversity due to climatic and anthropogenic factors in Hakaluki Haor, an ecologically critical wetland in northeast Bangladesh. Heliyon 7, e05861. https:// doi.org/10.1016/j.heliyon.2020.e05861.

Bahadur A.S.M.S. 2004. Production and Marketing of cultured fish in selected areas of Bangladesh. An unpublished thesis submitted to the faculty of Agricultural Economics and Rural sociology, BAU, Mymensingh. 99p.

BBS 2017. Statistical yearbook of Bangladesh, Bangladesh Bureau of Statistics, Government of Bangladesh, Bangladesh.

BFSA 2019. Bangladesh Food Safety Authority Ministry of Food, Govt. of Bangladesh Dhaka.

Bhuiyan A.L. 1964. Fishes of Dacca. Asiat. Soc. Pakistan. Dacca. Publn.No. 13, Pp.148.

CHOWDHURY M.M. AND IQUBAL, K.F. 2007. A survery on the availability of fish species in the landing centers of Dhaka city in Bangladesh. Bangladesh J. Zool. 35(2), Pp.259-267.

CUTER D., MILLER G. 2005. The role of public health improvements in health advances: The twentieth century United States. Demography (42)1-22, https://doi.org/10.1353/dem.2005.0002

DOF (Department of Fisheries) 2013. Fish week Compendium, Department of Fisheries, Ministry of Fisheries and Livestock, Government of the People's Republic Bangladesh, Dhaka.144p.

DOF (Department of Fisheries) 2018. Yearbook of Fisheries Statistics of Bangladesh, 2017-18. Fisheries Resources Survey System (FRSS), Department of Fisheries. Bangladesh, 35, 129.

FISHERIES STATISTICAL YEARBOOK (2017-18) Fisheries Resources Survey System, Department of Fisheries Bangladesh, Ministry of Fisheries and Livestock, Government of the People's Republic of Bangladesh, Ramna,Dhaka, Pp. 12.

FRSS 2016. Fisheries resources survey system (FRSS), fisheries statistical report of Bangladesh, vol. 32, Department of Fisheries, Bangladesh (2016), pp. 1-57.

HASAN M, ALAM J, AKTER O.R., KARIM M. 2014. An infrastructural analysis of the selected wholesale fish markets in Dhaka City, Bangladesh. World Journal of Fish and Marine Sciences; 6(2): 142-145.

HUSSAIN M.M., UDDIN M.H. 1995. Quality Control and Marketing of Fish and Fish Products: Needs for Infrastructure and Legal Support. Pp. 233-272.

ISLAM MD.S., KHABIR UDDIN M., TAREQ S.M., SHAMMI M., IBNE KAMAL A., SUGANO T., KURASAKI M., SAITO T., TANAKA S., KURAMITZ H. 2015. Alteration of Water pollution level with the seasonal changes in mean daily discharge in three main rivers around Dhaka City, Bangladesh. Environments 2, 280-294. http://dx.doi.org/10.3390/environments2030280. 
Jessica R.B., Shakuntala H.T., Geoffrey C.M., Md. Abdul W., Mostafa A.R.H, Jette J., James S. 2015. Nutrient composition of important fish species in Bangladesh and potential contribution to recommended nutrient intakes, Journal of Food Composition and Analysis, 42, 120-133.

RAHMAN A.K.A. 2005. Freshwater fishes of Bangladesh. 2nd ed., Zool. Soc. Bangladesh, Dhaka, Bangladesh, xvii, $394 \mathrm{Pp}$.

RAHMAN A.K.A.1997. Fish Marketing in Bangladesh: Status and Issue. The University Press Ltd. Dhaka, Bangladesh. Pp. 99-114.

RAHMAN M.M., HOSSAIN M.M., RAHMAN S.M., ALAM M.H. 2009. Fish Marketing System in Khulna, Bangladesh.j.innov.dev.Pp. 27-31.

RAJTS F AND SHELLEY CC. 2020. Guide to improving live fish transportation with special attention to COVID-19 pandemic in Bangladesh and other tropical developing countries. Penang, Malaysia: WorldFish.

YEASMIN S., LATIFA G.A. AND CHOWDHURY G.W. 2017. Diversity of ichthyofauna of Feni and Muhuri rivers, Feni, Bangladesh. Bangladesh Journal of Zoology, 45: 47-60.

(Manuscript received on 10 April 2020 revised on 22 August, 2021) 